\title{
Expression of annexin II and stromal tenascin C promotes epithelial to mesenchymal transition and correlates with distant metastasis in pancreatic cancer
}

\author{
NAOKO YONEURA, SHIGETSUGU TAKANO, HIDEYUKI YOSHITOMI, YASUYUKI NAKATA, \\ REIRI SHIMAZAKI, SHINGO KAGAWA, KATSUNORI FURUKAWA, TSUKASA TAKAYASHIKI, \\ SATOSHI KUBOKI, MASARU MIYAZAKI and MASAYUKI OHTSUKA
}

Department of General Surgery, Chiba University, Graduate School of Medicine, Chiba 260-8677, Japan

Received December 8, 2017; Accepted April 30, 2018

DOI: $10.3892 /$ ijmm.2018.3652

\begin{abstract}
The interaction between cancer cells and stromal components contributes to cancer invasion and metastasis in pancreatic ductal adenocarcinoma (PDAC). The present study investigated the role of the correlation between annexin II (ANX2) and stromal tenascin C (TNC) with the progression of PDAC. The functions of the expression ANX2 and TNC were assessed in in vitro experiments using mouse and human PDAC cells, and the clinical effect was analyzed using immunohistochemistry with surgically resected PDAC tissues. The effects on epithelial to mesenchymal transition (EMT), invasion, putative cancer stemness, and anoikis resistance were examined in vitro using murine precancerous pancreatic intraepithelial neoplasia (PanIN) cells and murine and human invasive PDAC cells with ANX2 knockdown using specific small interfering RNA (siRNA)s and recombinant TNC (rTNC). ANX2 was expressed at a high level in primary PanIN cells and invasive PDAC cells, compared with the levels in liver metastatic PDAC cells. In the ANX2-knockdown cells, there were fewer cells with a morphological mesenchymal appearance in three-dimensional culture and invasion was reduced compared with that in the control cells. Morphological change into the mesenchymal phenotype and invasion were enhanced by rTNC treatment in the control PDAC cells but not in the ANX2-knockdown cells. Pancreatosphere formation assays
\end{abstract}

Correspondence to: $\mathrm{Dr}$ Shigetsugu Takano, Department of General Surgery, Chiba University, Graduate School of Medicine, 1-8-1, Inohana, Chuo-ku, Chiba 260-8677, Japan

E-mail: stakano@faculty.chiba-u.jp

Abbreviations: ANX2, annexin II; TNC, tenascin C; PanIN, pancreatic intraepithelial neoplasia; PDAC, pancreatic ductal adenocarcinoma; EMT, epithelial to mesenchymal transition; CSC, cancer stem cell; IHC, immunohistochemistry; DFS, disease-free survival; OS, overall survival

Key words: annexin II, tenascin C, pancreatic cancer, epithelial to mesenchymal transition, invasion, distant metastasis showed that ANX2 and TNC facilitated the maintenance of stem-like characters in PDAC cells. Furthermore, anoikis assays indicated that the interaction of ANX2-TNC contributed to anoikis resistance in PDAC cells. In the immunohistochemistry analyses, the group with a high expression of ANX2 and high stromal TNC was significantly correlated with distant metastasis, and was associated with hematogenous/peritoneal recurrence and poor outcomes following surgery in resected human primary PDAC tissues. In conclusion, the results demonstrated that ANX2 and stromal TNC regulated invasion in addition to stemness and anoikis resistance, which are crucial for metastasis in the progression of PDAC. These results indicate the potential of the ANX2-TNC axis as a therapeutic target for PDAC metastasis.

\section{Introduction}

Pancreatic ductal adenocarcinoma (PDAC) is an aggressive and devastating form of cancer (1), and it is expected to become the second leading cause of cancer-associated mortality by 2030 in the United States (2). Despite advances in multidisciplinary treatments for PDAC, improving clinical outcomes has proven difficult. The major reasons for this lack in improvement are associated with the difficulties in providing an early diagnosis, the high malignant potential in terms of invasiveness and metastasis, and resistance against chemoradiotherapy (3). Therefore, the elucidation of the molecular mechanism involved in the progression of PDAC is urgently required.

Annexins comprise a family of calcium-dependent phospholipid-binding proteins with diverse cellular functions, including membrane-cytoskeleton organization and regulation of ion channel activity (4). Diaz indicated that tissue plasminogen activator (t-PA), which is overexpressed in PDAC cells, binds specifically to annexin II (ANX2), a member of the annexin family, on the extracellular membrane of PDAC cells, leading to the activation of tumor cell invasion (5). It has also been reported that ANX2 contributes to the epithelial to mesenchymal transition (EMT) of PDAC cells and liver metastasis in mouse PDAC models (6). In our previous study, ANX2 was identified as a gemcitabine-resistant factor in PDAC using a comprehensive proteomics technique (7), and it was revealed 
that the Akt/mammalian target of rapamycin signaling pathway is involved in the mechanisms of gemcitabine resistance induced by ANX2 in PDAC cells (8). Therefore, ANX2 has diverse functional roles and is likely a crucial factor in the progression of PDAC.

A dense stromal desmoplastic reaction surrounding cancer cells is a hallmark histological feature in PDAC (3). The stromal components consist of pancreatic stellate cells, cancer-associated fibroblasts, macrophages, infiltrating immune cells, and extracellular matrix (ECM), which orchestrate with cancer cells and are crucial in cancer progression (9). These interactions between cancer cells and the stroma in PDAC carcinogenesis, metastasis and therapeutic resistance have been thoroughly investigated using several approaches. Tenascin $\mathrm{C}$ (TNC) is one of the major ECM components that accelerates the invasion and metastasis of cancer cells $(10,11)$. It has been reported that TNC directly binds to ANX2 in PDAC cells (12); however, the molecular functions induced by the interaction between ANX2 and stromal TNC in PDAC progression remain to be elucidated.

The present study investigated the functional roles of the interaction of ANX2 with stromal TNC in the progression of PDAC. The interaction of these molecules drives pre-invasive pancreatic intraepithelial neoplasia (PanIN) cells to transition to the mesenchymal phenotype, and accelerates their self-renewal capacity and anoikis resistance to induce metastasis. The findings of the present study provide an improved understanding of the functional effects induced by the interaction of these two molecules in the tumor microenvironment of PDAC, which may assist in the development of a novel therapeutic target for PDAC.

\section{Materials and methods}

Patients and human tissue samples. PDAC tissues were obtained from 78 consecutive patients who underwent R0 (no residual tumor) or R1 (microscopic residual tumor) surgical resection in the Department of General Surgery, Chiba University Hospital (Chiba, Japan) between January 2006 and December 2010. All patients were diagnosed with primary PDAC histologically. The Ethics Committees of Chiba University, Graduate School of Medicine (Chiba, Japan) approved the protocol of the present study, and written informed consent was obtained from each patient prior to surgery.

Murine and human pancreatic cell lines and culture conditions. The murine PanIN cells (KC), invasive PDAC cells (KPC1 and KPC2), and liver metastatic PDAC cells (KPCLiv) were kindly provided by Dr Sunil Hingorani (University of Washington, Seattle, WA, USA) (13). In brief, each cell line was isolated from a murine PanIN lesion (Pdxl-cre; LSL-Kras $\left.{ }^{G 12 D /+}\right)$ for $\mathrm{KC}$, a primary PDAC in KPC mice (Pdxl-cre; LSL-Kras ${ }^{G 12 D /+}$; $p 53^{R 172 H /+}$ ) for KPC1 and KPC2, and a liver metastatic lesion in KPC mice (Pdxl-cre; LSL-Kras ${ }^{G 12 D /+} ;$ p53 $3^{R 172 H /+}$ ) for KPCLiv. The PANC-1 human PDAC cell line was obtained from the American Type Culture Collection (Manassas, VA, USA). All cell lines were cultured in Dulbecco's modified Eagle's medium (DMEM; Sigma-Aldrich; EMD Millipore, Billerica, MA, USA) with $10 \%$ fetal bovine serum (Thermo Fisher
Scientific, Inc., Waltham, MA, USA) and antibiotics (1\% penicillin and streptomycin).

Western blot analysis. The proteins were extracted from the above cultured cells with RIPA buffer (Sigma-Aldrich; EMD Millipore). Each protein sample (20-50 $\mu \mathrm{g})$ was lysed in buffer (Laemmli Sample Buffer; Bio-Rad Laboratories, Inc., Hercules, CA, USA) containing 5\% 2-mercaptoethanol and incubated for $10 \mathrm{~min}$ at $97^{\circ} \mathrm{C}$. Following the measurement of the protein concentration of each sample using Pierce ${ }^{\mathrm{TM}}$ BCA Protein Assay kit (Thermo Fisher Scientific, Inc.), $30 \mu \mathrm{g}$ of proteins were then separated by electrophoresis on $5-12.5 \%$ XV PANTERA Gels (DRC, Tokyo, Japan) and transferred onto a membrane (PerkinElmer, Inc., Waltham, MA, USA). The membranes were blocked in 5\% skim milk diluted with $0.1 \%$ Tris-buffed saline with Tween-20 at room temperature for $60 \mathrm{~min}$. The membranes were then incubated with the following primary antibodies overnight at $4^{\circ} \mathrm{C}$ : Anti-ANX2 polyclonal antibody (1:10,000 dilution; cat. no. 11256-1-AP; ProteinTech Group, Inc., Rosemont, IL, USA), anti-TNC monoclonal antibody (1:1,000 dilution; cat. no. 12221; Cell Signaling Technology, Inc., Danvers, MA, USA), anti-E-cadherin polyclonal antibody (1:1,000 dilution; cat. no. sc-7870; Santa Cruz Biotechnology, Inc., Santa Cruz, CA, USA), anti-vimentin polyclonal antibody (1:1,000 dilution; cat. no. 3932; Cell Signaling Technology, Inc.), and anti- $\beta$-actin monoclonal antibody (1:2,000 dilution; cat. no. 5125; Cell Signaling Technology, Inc.). Subsequently, the membranes were incubated at room temperature for $60 \mathrm{~min}$ with anti-rabbit $\mathrm{IgG}$ horseradish peroxidase secondary antibody (1:2,000 dilution; cat. no. sc-2301; Santa Cruz Biotechnology, Inc.), in blocking buffer. The membranes were then incubated with enhanced chemiluminescence detection reagent $\left(A m e r s h a m^{\mathrm{TM}} \mathrm{ECL}^{\mathrm{TM}}\right.$ Prime western blotting detection reagent; GE Healthcare Life Sciences, Chalfont, UK) and developed with a LAS-4000UV mini luminescent image analyzer (Fujifilm, Tokyo, Japan). The intensity of each band was quantified by densitometric analysis using ImageJ software version 1.51 (National Institutes of Health, Bethesda, MD, USA) and used to calculate the relative protein level normalized to $\beta$-actin.

Small interfering RNAs (siRNAs) and reagents. siRNAs specifically targeting mouse ANX2 mRNA (m-siANX2) and human ANX2 mRNA (h-siANX2) to inhibit the expression of ANX2 were purchased, including double-stranded synthetic siANX2s; m-siANX2-1 SASI_Mm01_00187632: Forward, 5'-GCAAGUCCCUGUACUACUATT-3'; Reverse, 5'-UAG UAGUACAGGGACUUGCTT-3'; m-siANX2-2 SASI_ Mm01_00187634: Forward,5'-GUAUGAUGCUUCGGAACU ATT-3'; Reverse, 5'-UAGUUCCGAAGCAUCAUACTT-3'; h-siANX2-1 SASI_Hs01_00246294: Forward, 5'-GUUACA GCCCUUAUGACAUTT-3', Reverse, 5'-AUGUCAUAAGGG CUGUAACTT-3'; h-siANX2-2 SASI_Hs01_00246296: Forward, 5'-GAACUUGCAUCAGCACUGATT-3'; Reverse, 5'-UCAGUGCUGAUGCAAGUUCTT-3' (Sigma-Aldrich; EMD Millipore), and sicontrol All Stars negative control siRNA (Qiagen, Inc., Valencia, CA, USA). The cells $\left(1 \times 10^{5}\right)$ were cultured for $24 \mathrm{~h}$, and were then transfected with siRNAs (30 nmol/1 final concentration) in Opti-MEM I reduced serum medium (Thermo Fisher Scientific, Inc., Waltham, 
MA, USA) using Lipofectamine ${ }^{\mathrm{TM}}$ RNAiMAX transfection reagent (Invitrogen; Thermo Fisher Scientific, Inc.). At $72 \mathrm{~h}$ post-siRNA transfection, whether knockdown was sufficient was evaluated using western blot analysis. The cells were used for the subsequent assays at $24 \mathrm{~h}$ post-transfection.

Recombinant TNC (rTNC) treatment. The rTNC (TNC purified protein; EMD Millipore) was added to the culture medium at room temperature at $2.5 \mu \mathrm{g} / \mathrm{ml}$ for western blot analysis, 3D cell culture and the pancreatosphere formation assay, and at $5.0 \mu \mathrm{g} / \mathrm{ml}$ for the invasion assay.

Three-dimensional (3D) organotypic cell culture system. 3D organotypic pancreatic cell culture was performed as previously described $(14,15)$. Briefly, the cells transfected with siANX2s or sicontrol were suspended in collagen solution with or without $\mathrm{rTNC}(2.5 \mu \mathrm{g} / \mathrm{ml}$ final concentration). The collagen-coated cells $(1,250$ cells/well) were poured into 4-well chamber slides (Thermo Fischer Scientific, Inc.). The culture medium containing rTNC was added to each well on a collagen layer, followed by culture with replacement of the medium every 3 days. After 7 days, images of the cells were captured using AxioVision (version 4.3; Carl Zeiss AG, Oberkochen, Germany). The morphology of cells was classified into three types: Spheroid cysts, irregular cysts, and spindle-shaped cells; and their numbers were observed and counted as previously described (16).

Cell invasion assay. Cell Biolabs CytoSelect ${ }^{\mathrm{TM}}$ 24-eell cell invasion assay kits (Cell Biolabs, San Diego, CA, USA) utilizing basement membrane-coated inserts were used according to the manufacturer's protocol. In brief, cells transfected with siANX2s or sicontrol were suspended in serum-free medium. Following overnight starvation, the cells were seeded at $3.0 \times 10^{5}$ cells/well in the upper chamber and incubated with medium containing serum with/without $5.0 \mu \mathrm{g} / \mathrm{ml} \mathrm{rTNC}$ in the lower chamber for $48 \mathrm{~h}$. The invasive cells passing through the basement membrane layer were stained and quantified at an optical density (OD) of $595 \mathrm{~nm}$ in a plate reader (iMark ${ }^{\mathrm{TM}}$ microplate reader; Bio-Rad Laboratories, Inc.) following extraction.

Pancreatosphere formation assay. Pancreatosphere formation assays were performed as described previously $(16,17)$. Briefly, the cells transfected with siANX2s or sicontrol in sphere medium with or without $2.5 \mu \mathrm{g} / \mathrm{ml} \mathrm{rTNC}$ were seeded at 15 cells/well in 96-well ultra-low attachment plates (Corning, Inc., Corning, NY, USA). Following 7 days of incubation at $37^{\circ} \mathrm{C}$, the cells were evaluated and the number of spheres with a diameter $>50 \mu \mathrm{m}$ were counted using an inverted microscope (Axio Observer Z1; Carl Zeiss AG, Oberkochen, Germany). The sphere formation rate was assessed as the percent increase in the number of spheres on day 7 with respect to the number of spheres observed on day 1 .

Anoikis assay. To assess anoikis resistance, which is the resistance for apoptosis following loss of contact with the ECM, an anoikis assay was performed as described previously (18). Briefly, the cells were continuously rotated on tube rotator (HB-1000 Hybridizer hybridization oven;
Ultra-Violet Products, Inc., Upland, CA, USA) for $24 \mathrm{~h}$. Subsequently, 3,000 cells/well suspended in medium with $0.3 \%$ agar were seeded onto 24-well culture plates coated with a lower layer of medium with $1 \%$ agar, and were cultured for 14 days at $37^{\circ} \mathrm{C}$. KPC cells $(2,000 / \mathrm{ml})$ transfected with siANX2s or sicontrol in medium with or without $2.5 \mu \mathrm{g} / \mathrm{ml}$ rTNC were incubated in medium without growth factor with rotation for $24 \mathrm{~h}$ at $37^{\circ} \mathrm{C}$. The colony formation assay was then performed (16). The number of colonies was determined at 14 days following cell seeding. Tumor colonies were visualized following staining with Giemsa stain solution (dilution 1:20, Waki Pure Chemical Industries, Ltd., Osaka, Japan).

Immunohistochemistry (IHC). Formalin-fixed paraffinembedded tissue samples were cut into $4-\mu \mathrm{m}$-thick slices and deparaffinized. The antigens were activated by autoclaving the tissue slides in citric acid buffer $(0.01 \mathrm{~mol} / 1, \mathrm{pH} 6.0)$ at $120^{\circ} \mathrm{C}$ for $10 \mathrm{~min}$. The slides were blocked with hydrogen peroxide $\left(\mathrm{H}_{2} \mathrm{O}_{2}\right)$ diluted to $3 \%$ with methanol for $15 \mathrm{~min}$ to inactivate endogenous peroxidase. Immunohistochemical staining was performed using the hyper-sensitive polymer method (Dako EnVision+ kit; Dako; Agilent Technologies $\mathrm{GmbH}$; Agilent Technologies GmbH, Waldbronn, Germany) for ANX2 and using the labeled streptavidin-biotin-peroxidase method (Dako; Agilent Technologies GmbH LSAB2 kit; Dako; Agilent Technologies $\mathrm{GmbH}$ ) for TNC, according to the manufacturer's protocol. Following protein blocking, the slides were incubated with the following primary antibodies: Anti-ANX2 polyclonal antibody (1:200 dilution; cat. no. sc-9061; Santa Cruz Biotechnology, Inc.) and monoclonal anti-human TNC antibody (1:4,000 dilution; cat. no. T2551; Sigma-Aldrich; EMD Millipore) overnight at $4^{\circ} \mathrm{C}$. Counterstaining was performed with hematoxylin prior to dehydration, penetration and mounting. Using an inverted microscope (BX40; Olympus Corporation, Tokyo, Japan), the staining intensities of ANX2 and TNC were evaluated independently by two investigators with a pathologist. The ANX2 staining patterns were scored as previously described $(7,8)$ : Low expression, 0-30\% of cancer cells with cell surface staining; High expression, $>30 \%$ of cancer cells with cell surface staining. The TNC staining patterns were scored as follows: Low expression, no expression in the stroma surrounding the cancer; High expression, linear staining in the stroma surrounding the cancer. The staining intensity of normal pancreatic ductal cells was used as an internal positive control.

Statistical analysis. The correlations between ANX2/TNC staining expression and the characteristics of patients with PDAC were evaluated using the $\chi^{2}$ test. Survival rates were calculated using Kaplan-Meier analysis and assessed using the log-rank test. The in vitro experiments were performed at least three times independently and data were analyzed using Welch's t-test. $\mathrm{P}<0.05$ was considered to indicate a statistically significant difference. Values are expressed as the mean \pm standard error of the mean or standard deviation. The above series of statistical analyses were performed using JMP ${ }^{\circledR}$ PRO 13 software (SAS Institute Inc., Cary, NC, USA). 


\section{Results}

ANX2 is expressed at high levels in murine primary pancreatic neoplastic cells. The present study examined the protein expression levels of ANX2 and endogenous TNC among murine pancreatic neoplastic cells isolated from the pancreas of KC mice or pancreas/liver metastases of KPC mice, which are genetically engineered mouse models. The expression of ANX2 was upregulated in primary precancerous PanIN (KC) cells and invasive PDAC (KPC) cells, but was relatively downregulated in liver metastatic PDAC (KPCLiv) cells, compared with that in primary KPC cells (Fig. 1A). Endogenous TNC in the invasive KPC cells was expressed at a high level, compared with that in precancerous and liver metastatic pancreatic cells. The present study also assessed the correlation in protein expression between ANX2 and representative epithelial/mesenchymal markers, E-cadherin and vimentin, among these cell lines. There was no correlation in expression between ANX2 and E-cadherin/vimentin according to the western blot results.

Subsequently, the present study determined whether the expression of ANX2 affects the expression of endogenous TNC. ANX2 knockdown using ANX2-specific siRNAs did not affect the expression of TNC in KC or KPC1 cells (Fig. 1B). Subsequently, whether ANX2 with extrinsic TNC influences the expression of EMT markers in $\mathrm{KC}$ cells was determined. The exposure to rTNC resulted in the significant downregulation of E-cadherin in $\mathrm{KC}$ cells $(\mathrm{P}=0.025)$; however, there was no effect on the expression of E-cadherin in the ANX2-knockdown cells (Fig. 1C and D). Taken together, these data indicated that ANX2 and extrinsic TNC may induce pancreatic neoplastic cells to transition to the mesenchymal phenotype during the progression of PDAC.

ANX2-TNC interaction regulates mesenchymal morphology in pancreatic neoplastic cells. To examine the functional effects of ANX2 and TNC in pancreatic neoplastic cells, the present study first used a well-established 3D organotypic culture system. Based on the cellular phenotype, the cultured KC cells exhibited representative morphological characteristics, including spheroid cysts, irregular cysts, and a spindle shape, in the 3D culture system (Fig. 2A). To determine whether ANX2, TNC, or the ANX2-TNC interaction affects the morphological changes in pancreatic neoplastic cells, ANX2 knockdown and rTNC treatment were performed in KC cells. The ANX2-knockdown cells demonstrated significantly altered morphology and a smaller population of mesenchymal spindle-shaped cells, compared with the KC control cells (Fig. 2B). Additionally, it was observed that rTNC treatment resulted in a significant increase spindle-shaped cells in the control cells $(\mathrm{P}=0.001)$ but not in the ANX2-knockdown cells (Fig. 2B). 3D organotypic cell culture was also performed using a representative human PDAC cell line, PANC-1 cells. Western blot analysis confirmed that the expression of ANX2 in the PANC-1 cells was effectively suppressed by the specific siRNAs for human ANX2, and rTNC treatment did not affect the expression of ANX2 in PANC-1 cells (Fig. 2C). Consistent with the results in murine cells, the $3 \mathrm{D}$ cell culture experiments revealed that rTNC treatment increased the percentage of spindle-shaped cells in the control PANC-1 cells $(\mathrm{P}=0.029)$ but not in the ANX2-knockdown PANC-1 cells (Fig. 2D and E). These results suggested that ANX2 has a functional role in maintaining the mesenchymal phenotype and that the ANX2-TNC interaction fosters this in pancreatic neoplastic cells.

Interaction between ANX2 and TNC controls cell invasion in pancreatic neoplastic cells. The present study aimed to determine whether the ANX2-TNC interaction affects PDAC cell invasion, a representative mesenchymal feature. As previously reported, ANX2 knockdown resulted in significantly reduced invasion, compared with that observed in the control cells (m-sicontrol, vs. m-siANX2-1; $\mathrm{P}=0.008$, m-sicontrol, vs. m-siANX2-2; $\mathrm{P}=0.01$ ) (Fig. $3 \mathrm{~A}$ and $\mathrm{B}$ ). Notably, rTNC treatment significantly facilitated cell invasion in the KPC1 control cells but not in the ANX2-knockdown KPC1 cells $(P=0.039)$. Taken together, these results indicated that the ANX2-TNC interaction fosters cell invasion in the invasive PDAC cells.

ANX2 and TNC maintain stem-like characteristics in PDAC cells. To form metastatic colonies in a distant organ, self-renewal properties are required for the disseminated cancer cells. Pancreatosphere formation assays were performed in the present study to determine whether ANX2 confers the properties of putative cancer stem cells (CSCs) to KPC1 cells. In the pancreatosphere formation assays, ANX2 knockdown significantly reduced the number of sphere-forming cells, compared with that in the control KPC1 cells (m-sicontrol, vs. m-siANX2-1; $\mathrm{P}=0.008, \mathrm{~m}$-sicontrol, vs. m-siANX2-2; $\mathrm{P}=0.046$ ) (Fig. 4A and B). Subsequently, whether rTNC treatment also affected the self-renewal capacity of KPC1 cells was determined. Despite ANX2 knockdown, rTNC treatment increased the number of sphere-forming cells in the KPC1 cells. These results suggested that ANX2 and TNC have a functional role in the stem-like properties of PDAC cells, independently.

ANX2-TNC interaction contributes to anoikis resistance in PDAC cells. TNC is reported to accelerate the metastasis of cancer cells (11). Based on the association between the ANX2-TNC interaction and metastasis, it was hypothesized that the interaction may prevent cancer cells from anoikis following extravasation and subsequent colonization in metastatic site. To clarify this hypothesis in vitro, ANX2 knockdown was performed using the specific ANX2 siRNAs. An anoikis assay was performed to evaluate the potential role of the ANX2-TNC interaction in resistance to apoptosis following loss of contact from the ECM. Compared with that of the control KPC1 cells, the resistance of the ANX2-knockdown KPC1 cells to anoikis was significantly decreased (Fig. 4C and D). rTNC treatment significantly fostered colony formation in the KPC1 control cells but not in the ANX2-knockdown KPC1 cells $(\mathrm{P}=0.012)$. These results were also confirmed in human PANC-1 cells (Fig. 4E). These data suggested that ANX2 knockdown led to functionally impaired anchorage-independent growth and that the ANX2-TNC interaction accelerated colony formation in vitro. 
A

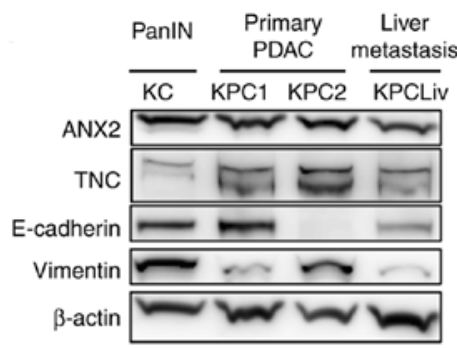

C

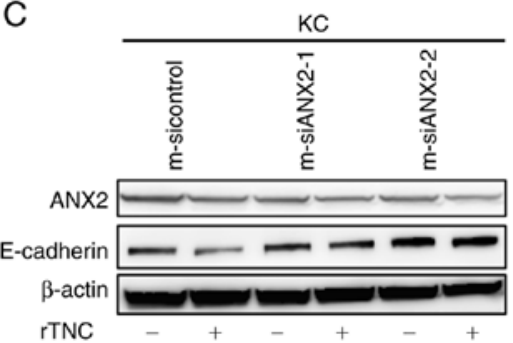

B

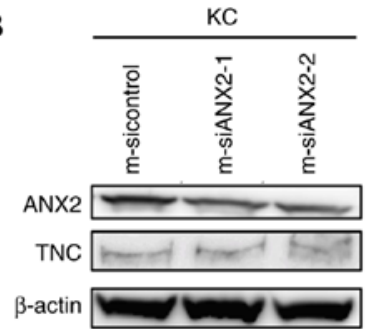

D

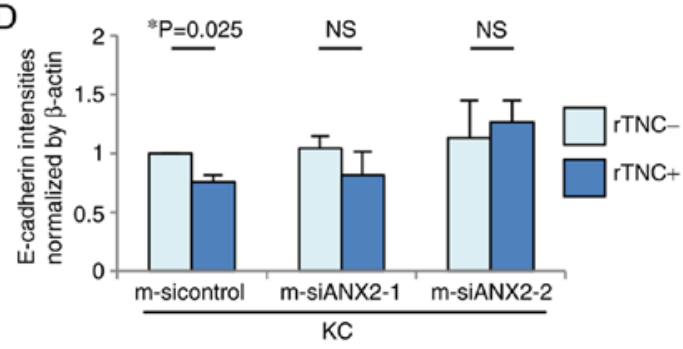

Figure 1. Differential expression of ANX2 and intrinsic TNC in murine pancreatic neoplastic cell lines. (A) Expression levels of ANX2 in various established murine pancreatic neoplastic cell lines derived from pancreatic intraepithelial neoplasia cells (KC), primary PDAC (KPC1 and KPC2) and PDAC liver metastases (KPCLiv) were determined by western blot analysis. (B) Expression levels of ANX2 and intrinsic TNC in KC and KPC1 cells treated with control and ANX2 siRNAs were determined by western blot analysis. (C) Comparison of expression levels of ANX2 and E-cadherin with or without rTNC treatment between KC cells treated with control and ANX2-specific siRNAs. (D) Comparative analysis of expression of E-cadherin with or without rTNC between KC cells treated with control and ANX2 siRNAs. The band intensities were normalized to that of $\beta$-actin. Experiments were performed at least three times. Error bars represent the standard the error of mean. PanIN, pancreatic intraepithelial neoplasia PDAC, pancreatic ductal adenocarcinoma; ANX2, annexin II; TNC, tenascin C; siRNA/si, small interfering RNA; rTNC, recombinant TNC; NS, not significant.

A

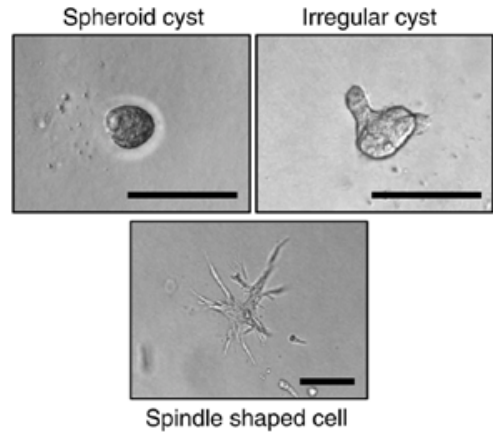

B

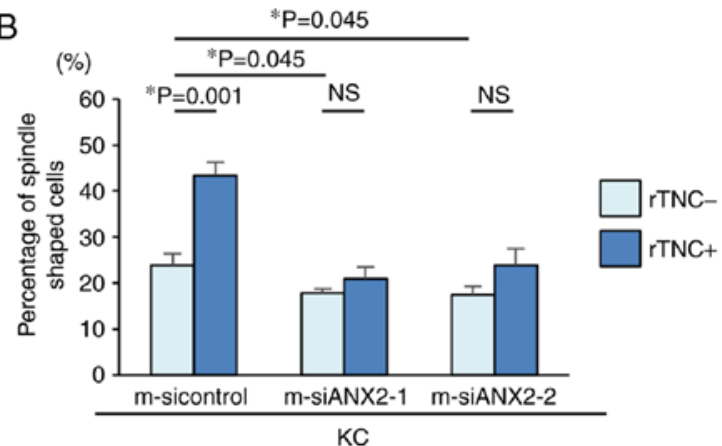

C

PANC-1

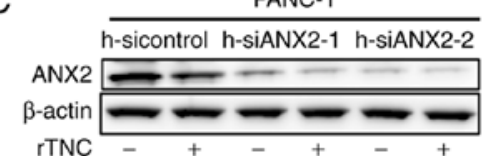

D

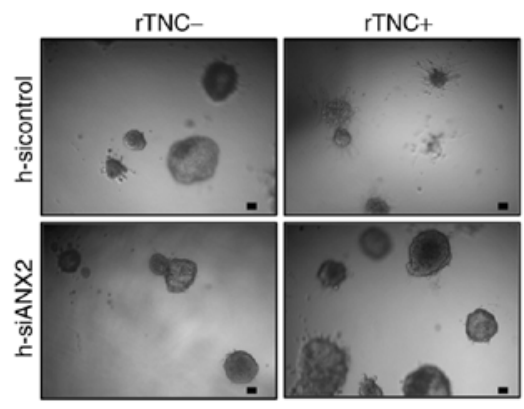

$\mathrm{E}$

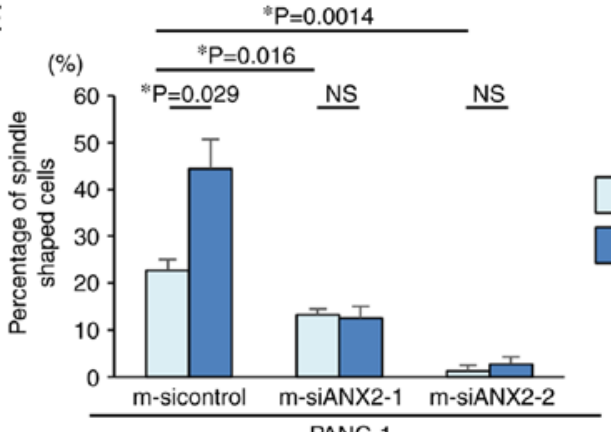

Figure 2. Morphological changes into the epithelial phenotype following ANX2 knockdown in precancerous KC cells. (A) Representative images of KC cells in 3D culture. Spheroid cyst (left panel), irregular cysts (right panel), and spindle shaped cells (lower panel). Scale bar=100 $\mu \mathrm{m}$. (B) Percentages of spindle-shaped cell formation in KC cells treated with control and ANX2 siRNAs with or without rTNC treatment. (C) Representative images of PANC-1 cells in 3D culture without (left panel) or with (right panel) rTNC treatment. Scale bar=100 $\mu \mathrm{m}$. (D) Comparative analysis of the expression of ANX2 with or without rTNC between PANC-1 cells treated with control and human ANX2 siRNAs. (E) Percentages of spindle-shaped cell formation in PANC-1 cells treated with control and ANX2 siRNAs with or without rTNC treatment. Error bars represent the standard error of the mean. ANX2, annexin II; siRNA/si, small interfering RNA; rTNC, recombinant tenascin C; 3D, three-dimensional; NS, not significant. 
A

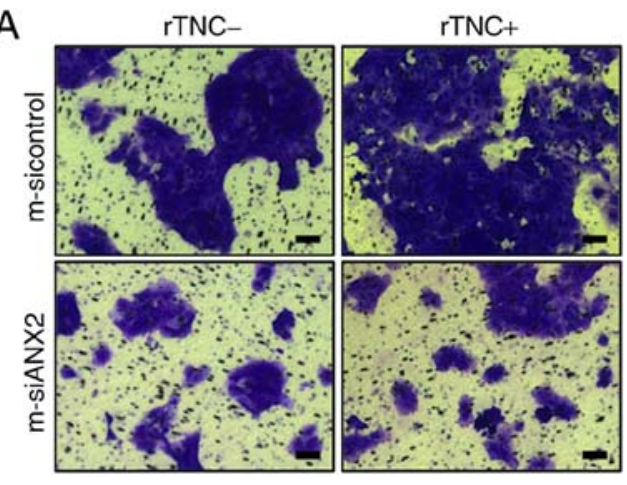

B

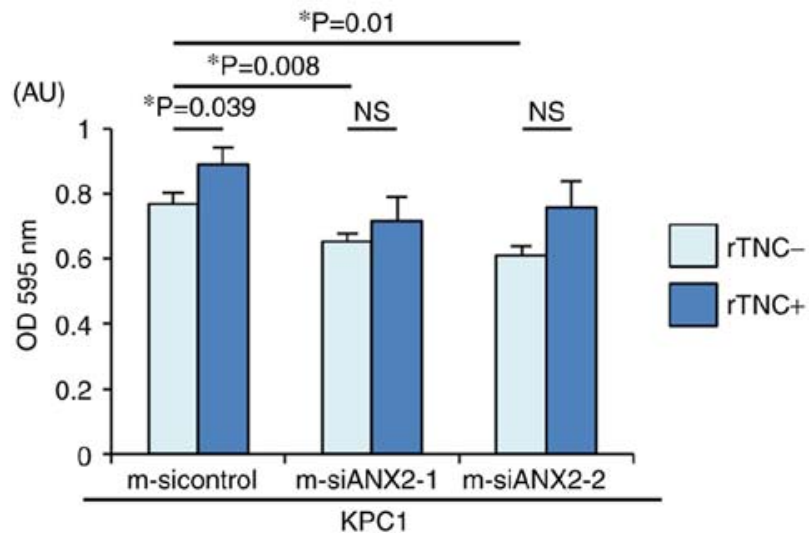

Figure 3. ANX2-TNC interaction accelerates pancreatic ductal adenocarcinoma cell invasion. (A) Representative images of invading KPC cells treated with control and ANX2 siRNAs with or without rTNC treatment following passage through the basement membrane layer. Scale bar=100 $\mu \mathrm{m}$. (B) Comparative analyses of invading KPC cells treated with sicontrol, siANX2-1, and siANX2-2 with or without rTNC treatment. Error bars represent the standard error of the mean. ANX2, annexin II; siRNA/si, small interfering RNA; rTNC, recombinant tenascin C; NS, not significant; OD, optical density.

A

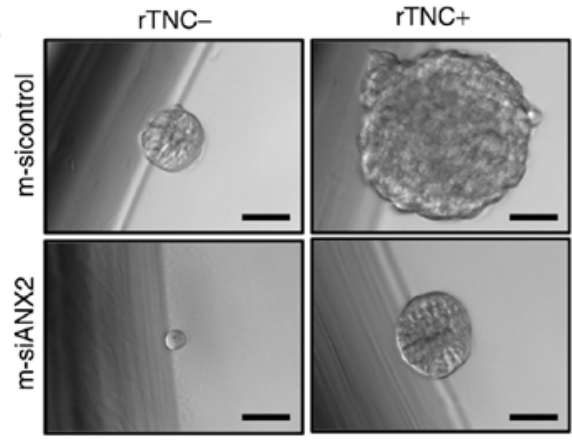

C

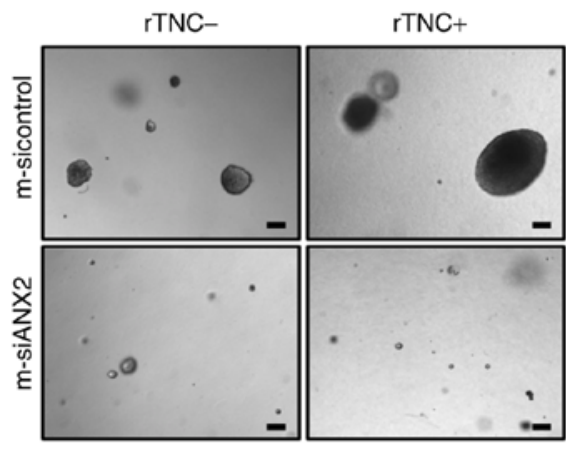

B

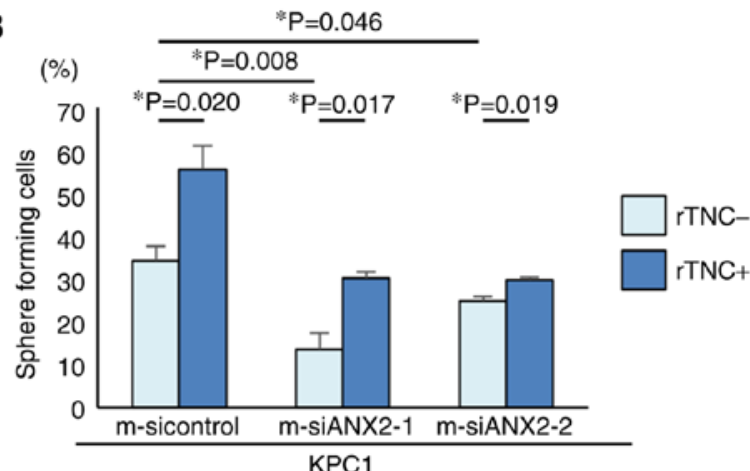

D

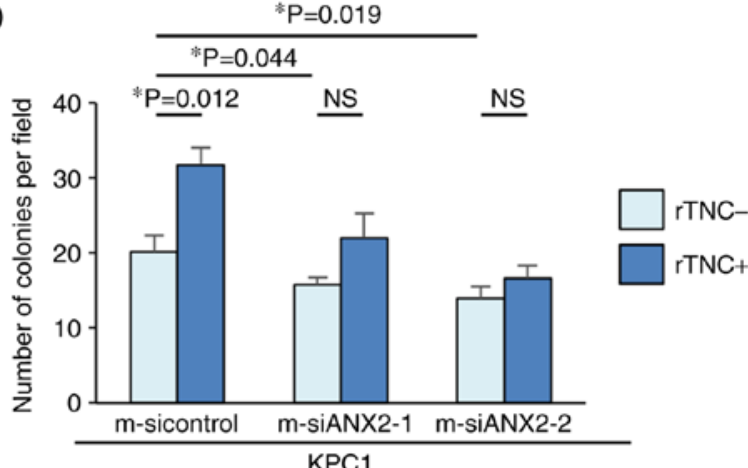

E

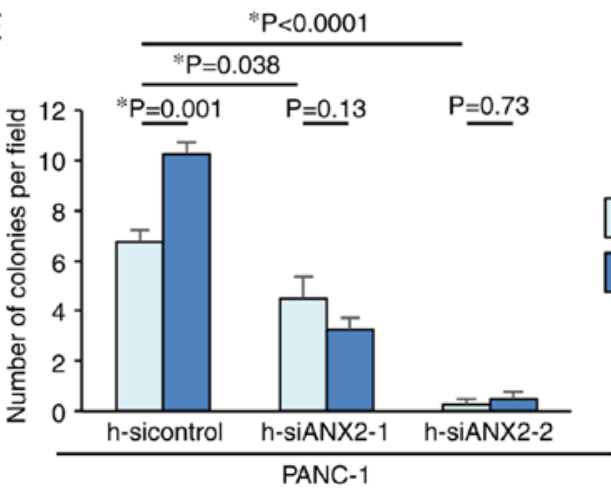

Figure 4. ANX2 and TNC enhance putative cancer stemness and the interaction fosters anoikis resistance in invasive pancreatic ductal adenocarcinoma cells. (A) Representative sphere formation in KPC1 cells without (left panel) or with (right panel) rTNC treatment. (B) Percentages of sphere forming cells were evaluated among the three KPC1 cells treated with siRNAs with or without rTNC treatment. (C) Representative colony formation in KPC1 cells without (left panel) or with (right panel) rTNC treatment. Numbers of colonies were evaluated among the three (D) KPC1 cells and (E) PANC-1 cells treated with siRNAs with or without rTNC treatment. Scale bar=100 $\mu \mathrm{m}$. Error bars represent the standard error of the mean. ANX2, annexin II; siRNA/si, small interfering RNA; rTNC, recombinant tenascin C; NS, not significant. 
A
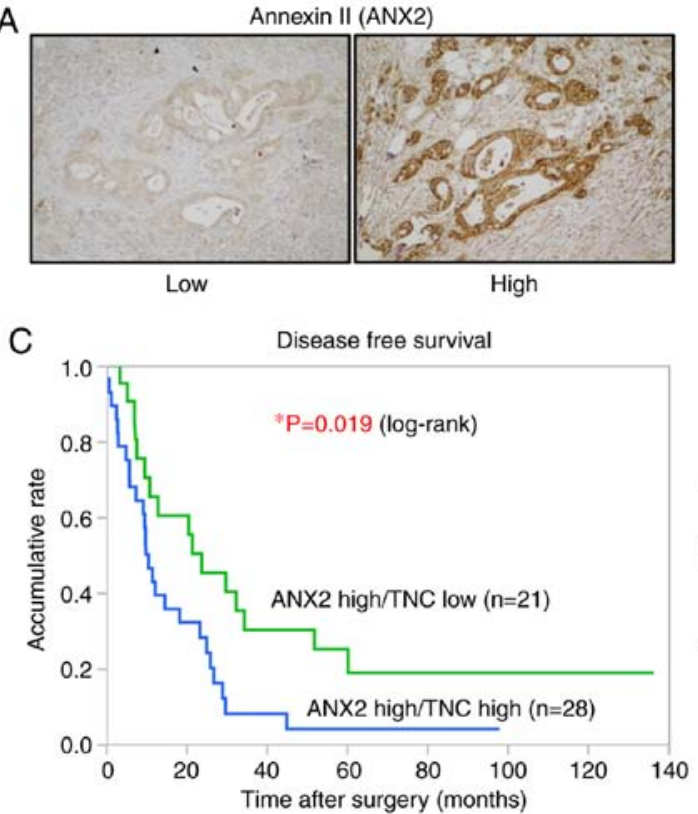

$\mathrm{B}$
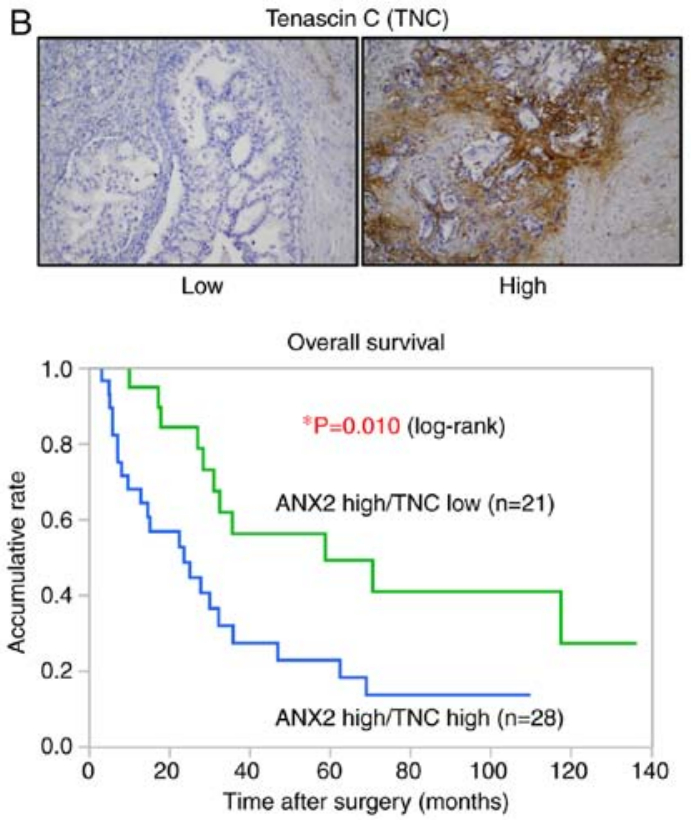

Figure 5. IHC analyses and correlation between ANX2 and stromal TNC in human PDAC samples. (A) Cell surface and cytoplasmic staining of ANX2 and (B) stromal staining of TNC in primary PDAC tissues were respectively categorized into Low expression (left panel) or High expression (right panel) groups based on the respective intensity criterions ( $\mathrm{n}=78$ ). Magnification, $\mathrm{x} 100$. (C) Green and blue lines indicate the ANX2 High/TNC Low and ANX2 High/TNC High groups, respectively. Kaplan-Meier survival curves show an unfavorable prognosis in the ANX2 High/TNC High group (DFS, P=0.019; OS, P=0.010, log-rank test). PDAC, pancreatic ductal adenocarcinoma; ANX2, annexin II; TNC, tenascin C.

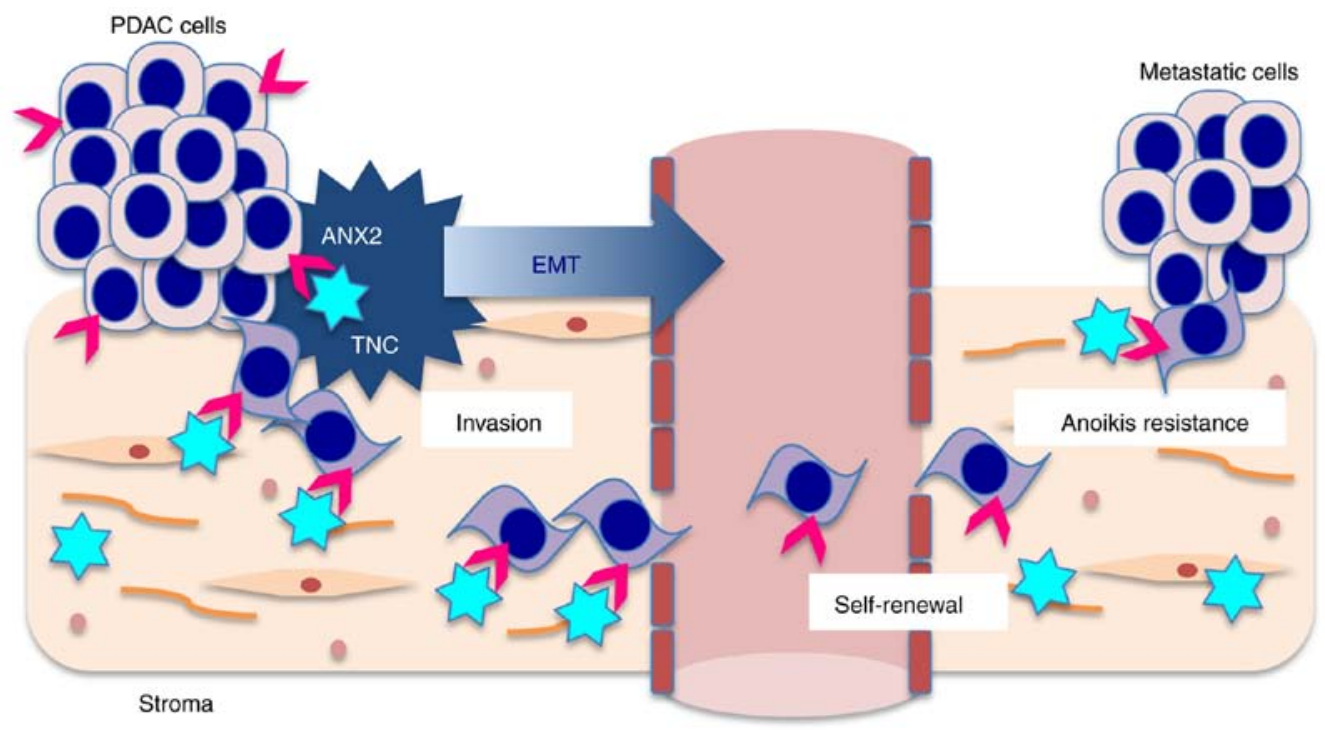

Figure 6. Schematic of the association between ANX2 and stromal TNC in the progression of PDAC. Pink arrows, ANX2 and blue stars, TNC. PDAC, pancreatic ductal adenocarcinoma; ANX2, annexin II; TNC, tenascin C; EMT, epithelial to mesenchymal transition.

High expression of ANX2 and stromal TNC positively correlates with distant metastasis in human PDAC. The present study examined the correlation between the expression of ANX2 and TNC and analyzed its clinical significance in PDAC. The expression levels of ANX2 and TNC in 78 resected human PDAC tissues were assessed by IHC staining. As previously shown, ANX2 was predominantly localized on the cell surface of PDAC cells $(7,8)$, whereas TNC was mainly expressed in the stroma surrounding ductal carcinoma cells (Fig. 5A and B). Among the 78, 49 cases (62.8\%) were classified as ANX2 High expression, and 29 cases (37.2\%) were classified as ANX2 Low expression based on the staining scores. The expression of TNC was also classified into the TNC high group (43 cases, 55.1\%) and TNC Low group ( 35 cases, $44.9 \%$ ) based on the staining scores. To evaluate the interaction between the expression of ANX2 and stromal TNC, all tissue samples were categorized into four groups as follows: 28 cases (35.9\%) in the ANX2 High/TNC High group, 21 cases (26.9\%) in the ANX2 High/TNC Low group, 15 cases (19.2\%) in the ANX2 Low/TNC High group, and 14 cases (17.9\%) in the ANX2 High/TNC Low group. No direct correlation between the expression of cancer cell surface ANX2 and stromal TNC was found in the primary PDAC tissues (Table I). 
Table I. Correlation between expression of ANX2 and stromal $\mathrm{TNC}$ in pancreatic ductal adenocarcinoma tissues.

\begin{tabular}{lccc}
\hline & \multicolumn{2}{c}{ Stromal TNC expression } & \\
\cline { 2 - 3 } Expression of ANX2 & High $(\mathrm{n}=43)$ & Low $(\mathrm{n}=35)$ & P-value \\
\hline High $(\mathrm{n}=49)$ & 28 & 21 & NS \\
Low $(\mathrm{n}=29)$ & 15 & 14 & \\
\hline
\end{tabular}

$\chi^{2}$ test was performed to determine statistical significance. ANX2, annexin II; TNC, tenascin C; NS, not significant.

High expression of ANX2 and TNC is associated with hematogenous and peritoneal recurrence following surgery and poor prognosis in human PDAC. Finally, to focus on the clinical significance of these proteins in PDAC, the present study analyzed the clinicopathological features of the stromal expression of TNC in the ANX2 High group. As shown in Table II, the expression of TNC was significantly associated with lymph node metastasis, distant metastasis and advanced stage in the ANX2 High group. A significant correlation was observed between hematogenous and peritoneal recurrence following curative surgery in the ANX2 High/TNC High group ( $\mathrm{P}=0.017, \chi^{2}$ test; Table III). Furthermore, the Kaplan-Meier analyses showed that patients with a high expression of TNC presented with significantly shorter disease-free survival (DFS) and overall survival (OS) rates, compared with those with a low expression of TNC in the ANX2 High group (DFS, $\mathrm{P}=0.019$; OS, $\mathrm{P}=0.010$, log-rank test; Fig. 5C). These clinical data suggested that the high expression of ANX2 and TNC is associated with hematogenous and peritoneal recurrence and poor prognosis in patients with PDAC.

\section{Discussion}

In the present study, it was demonstrated that ANX2 orchestrates with stromal TNC to induce EMT and promote distant metastasis in PDAC cells (Fig. 6). The in vitro data indicated that the interaction between ANX2 and extrinsic TNC facilitated pre-invasive PanIN cells to undergo EMT, and invasive PDAC cells to invade and metastasize. In clinical samples, IHC analyses also revealed that the ANX2 High/TNC High expression group was significantly correlated with distant metastasis, and was associated with hematogenous and peritoneal recurrence and poor prognosis following curative surgery.

The present study demonstrated that ANX2 was upregulated in mouse primary invasive PDAC cells, compared with the level in liver metastatic cells. To investigate the expression patterns of ANX2, intrinsic TNC and EMT markers, two PDAC cell lines (KPC1 and KPC2) were used, which originate from two different mice with the same mutant strains of KPC (Pdxl-cre; LSL-Kras $\left.{ }^{G 12 D /+} ; p 53^{R 172 H /+}\right)$. However, the expression pattern of EMT markers and morphology are different between these two cell lines. These results may reflect that cancer is heterogeneous in nature. Notably, ANX2 orchestrates with extrinsic TNC to induce EMT, which is considered an important process in the progression of PDAC from precancerous PanIN cells to invasive PDAC cells. Using a $3 \mathrm{D}$ cell culture system, the present study confirmed that ANX2 knockdown promoted the morphological changes from the epithelial to the mesenchymal phenotype in preinvasive $\mathrm{KC}$ cells. This finding indicated that ANX2 may be required to induce the mesenchymal phenotype in PDAC cells.

Invasion is a typical property of mesenchymal cells and a crucial step for metastasis $(19,20)$. Previous studies have demonstrated that ANX2 knockdown resulted in a significant reduction in cell invasion in various types of cancer cells, including PDAC cells $(5,21,22)$. Consistent with this, the present study demonstrated that ANX2 knockdown decreased PDAC cell invasion. Certain previous studies have indicated that EMT is not always essential for metastasis (23). However, cells undergoing EMT significantly contribute to recurrent lung metastasis formation following chemotherapy (24), and the suppression of EMT leads to an enhanced sensitivity to gemcitabine treatment (25). Supporting these findings, our previous study demonstrated that the expression of ANX2 was upregulated in gemcitabine-resistant PDAC cells (7), and the Akt/mammalian target of rapamycin signaling pathway was shown to be involved in the mechanism of chemoresistance conferred by ANX2 in PDAC cells (8).

TNC is a large glycoprotein located in the ECM, which regulates the interactions between the parenchyma and mesenchyme in conditions accompanying EMT and invasion $(26,27)$. TNC is overexpressed in PanIN lesions, and there is diffuse stromal expression in PDAC together with localization of its cell receptor ANX2 on the tumor cells (12). To determine whether the ANX2-TNC interaction is crucially involved in the invasion and metastasis of PDAC cells, the present study hypothesized that the interaction of ANX2 with stromal TNC can orchestrate to have functional roles and be a potential therapeutic target that regulates the invasion, putative CSC properties and anoikis resistance in the progression of PDAC. The present study observed that extrinsic TNC induced a decrease in the expression of E-cadherin, reflecting active EMT; furthermore, ANX2 knockdown reduced the morphological mesenchymal properties of murine PanIN KC cells. Therefore, these findings suggested that ANX2-TNC may cooperate in precancerous $\mathrm{KC}$ cells to induce the cells to undergo EMT during the progression of PDAC.

Oskarsson et al suggested that cancer cell-derived TNC remains essential for metastasis outgrowth until the tumor stroma takes over as a source of TNC in breast cancer (11). In the present study, it was also demonstrated that TNC enhanced the self-renewal capacity of PDAC KPC cells. Notably, independent of rTNC treatment, the expression of ANX2 also affected the self-renewal capacity of PDAC cells. It has been demonstrated that EMT-inducing transcription factors confer mesenchymal and stem cell properties (28). Using an anoikis assay, the present study examined the final step of the metastatic cascade, namely colony formation following extravasation from blood vessels, in in vitro experiments. Supportively, the anoikis assays clearly showed that the interaction of ANX2-TNC accelerated colony formation of murine and human PDAC cells. Considering these results, it may be possible that ANX2-TNC, in which ANX2 has sequential effects on inducing invasiveness and self-renewal capacity and TNC has effects in establishing a metastatic niche with CSC 
Table II. Clinicopathological features of stromal expression of TNC in patients with pancreatic ductal adenocarcinoma and high expression of ANX2.

\begin{tabular}{|c|c|c|c|c|}
\hline \multirow[b]{2}{*}{ Clinicopathological feature } & \multirow[b]{2}{*}{ Total $(n=49)$} & \multicolumn{2}{|c|}{ Stromal expression of TNC } & \multirow[b]{2}{*}{ P-value } \\
\hline & & $\operatorname{High}(\mathrm{n}=28)$ & Low $(n=21)$ & \\
\hline Age (years) & $66.3 \pm 8.6$ & $66.9 \pm 8.9$ & $66.3 \pm 8.5$ & NS \\
\hline Gender (male/female) & $24 / 25$ & $14 / 14$ & $10 / 11$ & NS \\
\hline Tumor size $(\mathrm{mm})$ & $28.4 \pm 8.4$ & $29.6 \pm 7.8$ & $26.6 \pm 9.1$ & NS \\
\hline$N(0 / 1)$ & $11 / 37$ & $3 / 24$ & $8 / 13$ & 0.026 \\
\hline $\mathrm{M}(0 / 1)$ & $43 / 6$ & $22 / 6$ & $21 / 0$ & 0.007 \\
\hline UICC stage (IIA $\geq / \geq I I B)$ & $10 / 39$ & $2 / 26$ & $8 / 13$ & 0.007 \\
\hline Residual tumor (R0/R1) & $35 / 14$ & $19 / 9$ & $16 / 5$ & NS \\
\hline
\end{tabular}

Data for age and tumor size are presented as the mean \pm standard deviation. $\chi^{2}$ test was performed to determine statistical significance. UICC, International Union Against Cancer; ANX2, annexin II; TNC, tenascin C; NS, not significant.

Table III. Correlation between stromal expression of TNC and recurrent form in the ANX2 High expression group.

\begin{tabular}{lcccc}
\hline & & \multicolumn{2}{c}{ Stromal expression of TNC } & \\
\cline { 3 - 4 } Recurrent form & Total $(\mathrm{n}=49)$ & High $(\mathrm{n}=28)$ & Low $(\mathrm{n}=21)$ & P-value \\
\hline Local $(-/+)$ & $26 / 23$ & $13 / 15$ & $13 / 8$ & NS \\
Lymph node $(-/+)$ & $45 / 4$ & $26 / 2$ & $19 / 2$ & NS \\
Hematogenous and peritoneal (-/+) & $23 / 26$ & $9 / 19$ & $14 / 7$ & 0.017 \\
\hline
\end{tabular}

$\chi^{2}$ test was performed to determine statistical significance. ANX2, annexin II; TNC, tenascin C; NS, not significant.

properties and anoikis resistance, facilitates metastasis during the metastatic cascade of PDAC.

In an analysis of clinical samples, a previous study showed that the co-overexpression of ANX2 and TNC was an independent factor of poor prognosis in patients with colorectal carcinoma (29). In the present study, the correlation between ANX2 and stromal TNC in human PDAC tissues was examined. Unexpectedly, there was no positive correlation between the expression of ANX2 and stromal TNC; however, it was confirmed that a high expression of both ANX2 and stromal TNC was significantly correlated with hematogenous/peritoneal recurrence and poor prognosis of patients with PDAC following curative surgery. Foley et al demonstrated that ANX2 promoted PDAC metastasis in vivo by knocking out ANX2 in KPC mice, a model that recapitulates the progression of human PDAC (30). Together with these data, the results of the present study suggested that the high expression of ANX2-TNC leads to poorer clinical outcomes for patients with PDAC.

In conclusion, the present study demonstrated that the ANX2-TNC interaction sequentially regulated EMT, invasion and metastatic colonization in the progression of PDAC. The ANX2-TNC partnership accelerated the progression of PDAC from invasion to metastasis. One of the limitations of the present study is that no gain-of-function or rescue experiments were performed. Further investigations are warranted to determine whether inhibition of the ANX2-TNC interaction influences the progression of PDAC in vivo and whether the ANX2-TNC axis may be a promising therapeutic target for PDAC.

\section{Acknowledgements}

The authors would like to thank Dr Suzuki and Dr Nishino (Department of General Surgery, Chiba University, Japan) for their technical support in experiments.

\section{Funding}

This study was supported by the Grant-in-Aid for Scientific Research (KAKENHI): KIBAN B (grant no. 15H04925 to ST, HY, SK and MM, and grant no. 17H04287 to MM, ST, HY, SK and MO); KIBAN C (grant no. 16K08979 to HY, ST, SK and MM, grant no. 16K10589 to MO, ST and SK, and grant no. $17 \mathrm{~K} 10661$ to SK and MO); the Challenge Exploratory Research (grant no. 16K15607 to ST, HY, SK and MM); and the Young Scientists B (grant no. 15K18439 to SK).

\section{Availability of data and materials}

The datasets used and/or analyzed during the current study are available from the corresponding author on reasonable request. 


\section{Authors' contributions}

NY contributed to the data acquisition, and drafting and revision of the manuscript. ST contributed to the study concept, experimental design, supervision and the data interpretation, and drafting, revision and finalization of the manuscript. YN and RS contributed to the data acquisition and analysis. SK, KF, TT and SK contributed to the conception of the study. HY, $\mathrm{MM}$ and MO contributed to the study concept and supervision.

\section{Ethics approval and consent to participate}

The Ethics Committees of Chiba University, Graduate School of Medicine approved the protocol of the present study, and written informed consent was obtained from each patient prior to surgery.

\section{Consent for publication}

Not applicable.

\section{Competing interests}

The authors declare that they have no competing interests.

\section{References}

1. Siegel RL, Miller KD and Jemal A: Cancer statistics, 2017. CA Cancer J Clin 67: 7-30, 2017.

2. Rahib L, Smith BD, Aizenberg R, Rosenzweig AB, Fleshman JM and Matrisian LM: Projecting cancer incidence and deaths to 2030: The unexpected burden of thyroid, liver, and pancreas cancers in the United States. Cancer Res 74: 2913-2921, 2014.

3. Ryan DP, Hong TS and Bardeesy N: Pancreatic adenocarcinoma. N Engl J Med 371: 1039-1049, 2014

4. Gerke V, Creutz CE and Moss SE: Annexins: Linking $\mathrm{Ca}^{2+}$ signalling to membrane dynamics. Nat Rev Mol Cell Biol 6: 449-461, 2005

5. Díaz VM, Hurtado M, Thomson TM, Reventós J and Paciucci R: Specific interaction of tissue-type plasminogen activator (t-PA) with annexin II on the membrane of pancreatic cancer cells activates plasminogen and promotes invasion in vitro. Gut 53: 993-1000, 2004.

6. Zheng L, Foley K, Huang L, Leubner A, Mo G, Olino K, Edil BH, Mizuma M, Sharma R, Le DT, et al: Tyrosine 23 phosphorylation-dependent cell-surface localization of annexin A2 is required for invasion and metastases of pancreatic cancer. PLoS One 6: e19390, 2011.

7. Takano S, Togawa A, Yoshitomi H, Shida T, Kimura F, Shimizu H, Yoshidome H, Ohtsuka M, Kato A, Tomonaga T, et al: Annexin II overexpression predicts rapid recurrence after surgery in pancreatic cancer patients undergoing gemcitabine-adjuvant chemotherapy. Ann Surg Oncol 15: 3157-3168, 2008.

8. Kagawa S, Takano S, Yoshitomi H, Kimura F, Satoh M, Shimizu H, Yoshidome H, Ohtsuka M, Kato A, Furukawa K, et al $\mathrm{Akt} / \mathrm{mTOR}$ signaling pathway is crucial for gemcitabine resistance induced by Annexin II in pancreatic cancer cells. J Surg Res 178: 758-767, 2012.

9. Neesse A, Algül H, Tuveson DA and Gress TM: Stromal biology and therapy in pancreatic cancer: A changing paradigm. Gut 64 1476-1484, 2015.

10. Lowy $\mathrm{CM}$ and Oskarsson $\mathrm{T}$ : Tenascin $\mathrm{C}$ in metastasis: $\mathrm{A}$ view from the invasive front. Cell Adh Migr 9: 112-124, 2015.

11. Oskarsson T, Acharyya S, Zhang XH, Vanharanta S, Tavazoie SF, Morris PG, Downey RJ, Manova-Todorova K, Brogi E and Massagué J: Breast cancer cells produce tenascin $\mathrm{C}$ as a metastatic niche component to colonize the lungs. Nat Med 17: 867-874, 2011.

12. Esposito I, Penzel R, Chaib-Harrireche M, Barcena U, Bergmann F, Riedl S, Kayed H, Giese N, Kleeff J, Friess H, et al Tenascin $\mathrm{C}$ and annexin II expression in the process of pancreatic carcinogenesis. J Pathol 208: 673-685, 2006.
13. Hingorani SR, Wang L, Multani AS, Combs C, Deramaudt TB, Hruban RH, Rustgi AK, Chang S and Tuveson DA: Trp53 $3^{R 172 H}$ and $\mathrm{Kras}^{G 12 D}$ cooperate to promote chromosomal instability and widely metastatic pancreatic ductal adenocarcinoma in mice. Cancer Cell 7: 469-483, 2005.

14. Wescott MP, Rovira M, Reichert M, von Burstin J, Means A Leach SD and Rustgi AK: Pancreatic ductal morphogenesis and the Pdx1 homeodomain transcription factor. Mol Biol Cell 20: 4838-4844, 2009.

15. Reichert M, Takano S, Heeg S, Bakir B, Botta GP and Rustgi AK: Isolation, culture and genetic manipulation of mouse pancreatic ductal cells. Nat Protoc 8: 1354-1365, 2013.

16. Takano S, Reichert M, Bakir B, Das KK, Nishida T, Miyazaki M, Heeg S, Collins MA, Marchand B, Hicks PD, et al: Prrx1 isoform switching regulates pancreatic cancer invasion and metastatic colonization. Genes Dev 30: 233-247, 2016.

17. Rovira M, Scott SG, Liss AS, Jensen J, Thayer SP and Leach SD: Isolation and characterization of centroacinar/terminal ductal progenitor cells in adult mouse pancreas. Proc Natl Acad Sci USA 107: 75-80, 2010.

18. Suzuki K, Takano S, Yoshitomi H, Nishino H, Kagawa S, Shimizu H, Furukawa K, Miyazaki M and Ohtsuka M: Metadherin promotes metastasis by supporting putative cancer stem cell properties and epithelial plasticity in pancreatic cancer. Oncotarget 8: 66098-66111, 2017.

19. Gupta GP and Massagué J: Cancer metastasis: Building a framework. Cell 127: 679-695, 2006.

20. Chaffer CL and Weinberg RA: A perspective on cancer cell metastasis. Science 331: 1559-1564, 2011.

21. Zhang F, Zhang L, Zhang B, Wei X, Yang Y, Qi RZ, Ying G, Zhang $\mathrm{N}$ and Niu R: Anxa2 plays a critical role in enhanced invasiveness of the multidrug resistant human breast cancer cells. J Proteome Res 8: 5041-5047, 2009.

22. Zhao P, Zhang W, Tang J, Ma XK, Dai JY, Li Y, Jiang JL, Zhang SH and Chen ZN: Annexin II promotes invasion and migration of human hepatocellular carcinoma cells in vitro via its interaction with HAb18G/CD147. Cancer Sci 101: 387-395, 2010.

23. Beerling E, Seinstra D, de Wit E, Kester L, van der Velden D, Maynard C,Schäfer R,vanDiestP, Voest E, vanOudenaarden A, etal: Plasticity between epithelial and mesenchymal states unlinks EMT from metastasis-enhancing stem cell capacity. Cell Rep 14: 2281-2288, 2016.

24. Fischer KR, Durrans A, Lee S, Sheng J, Li F, Wong S, Choi H, Rayes TE, Ryu S, Troeger J, et al: Epithelial-to-mesenchymal transition is not required for lung metastasis but contributes to chemoresistance. Nature 527: 472-476, 2015.

25. Zheng X, Carstens JL, Kim J, Scheible M, Kaye J, Sugimoto H, Wu CC, LeBleu VS and Kalluri R: Epithelial-to-mesenchymal transition is dispensable for metastasis but induces chemoresistance in pancreatic cancer. Nature 527: 525-530, 2015.

26. Chiquet-Ehrismann $\mathrm{R}$ and Chiquet M: Tenascins: Regulation and putative functions during pathological stress. J Pathol 200: 488-499, 2003.

27. Cai J, Du S, Wang H, Xin B, Wang J, Shen W, Wei W, Guo Z and Shen $X$ : Tenascin-C induces migration and invasion through JNK/c-Jun signalling in pancreatic cancer. Oncotarget 8: 74406-74422, 2017.

28. Mani SA, Guo W, Liao MJ, Eaton EN, Ayyanan A, Zhou AY, Brooks M, Reinhard F, Zhang CC, Shipitsin M, et al: The epithelial-mesenchymal transition generates cells with properties of stem cells. Cell 133: 704-715, 2008.

29. Emoto K, Yamada Y, Sawada H, Fujimoto H, Ueno M, Takayama T, Kamada K, Naito A, Hirao S and Nakajima Y: Annexin II overexpression correlates with stromal tenascin-C overexpression: A prognostic marker in colorectal carcinoma. Cancer 92: 1419-1426, 2001.

30. Foley K, Rucki A A, Xiao Q, Zhou D, Leubner A, Mo G, Kleponis J, Wu AA, Sharma R, Jiang Q, et al: Semaphorin 3D autocrine signaling mediates the metastatic role of annexin A2 in pancreatic cancer. Sci Signal 8: ra77, 2015. International (CC BY-NC-ND 4.0) License. 